FERMILAB-Conf-99/089

\title{
Large Acceptance Muon Storage Rings for Neutrino Production: Lattice Design
}

\author{
C. Johnstone and B. Autin \\ Fermi National Accelerator Laboratory \\ P.O. Box 500, Batavia, Illinois 60510
}

September 1999

Presented at PAC99, Particle Accelerator Conference, New York, New York, March 29-April 2, 1999 


\section{Disclaimer}

This report was prepared as an account of work sponsored by an agency of the United States Government. Neither the United States Government nor any agency thereof, nor any of their employees, makes any warranty, expressed or implied, or assumes any legal liability or responsibility for the accuracy, completeness, or usefulness of any information, apparatus, product, or process disclosed, or represents that its use would not infringe privately owned rights. Reference herein to any specific commercial product, process, or service by trade name, trademark, manufacturer, or otherwise, does not necessarily constitute or imply its endorsement, recommendation, or favoring by the United States Government or any agency thereof. The views and opinions of authors expressed herein do not necessarily state or reflect those of the United States Government or any agency thereof.

\section{Distribution}

Approved for public release; further dissemination unlimited.

\section{Copyright Notification}

This manuscript has been authored by Universities Research Association, Inc. under contract No. DE-AC02-76CH03000 with the U.S. Department of Energy. The United States Government and the publisher, by accepting the article for publication, acknowledges that the United States Government retains a nonexclusive, paid-up, irrevocable, worldwide license to publish or reproduce the published form of this manuscript, or allow others to do so, for United States Government Purposes. 


\title{
Large Acceptance Muon Storage Rings for Neutrino Production: Lattice Design *
}

\begin{abstract}
C. Johnstone, ${ }^{\dagger}$, FNAL, Batavia, IL 60510, B. Autin,CERN, Geneva, Switzerland

\section{Abstract}

The possibility of achieving the high muon fluxes suggested in recent work on muon colliders has revived interest in the idea of using muon storage rings for neutrino production. Through proper design of the lattice, a significant fraction of the stored muons can be converted into an intense, lowdivergence beam of neutrinos. This work examines the incorporation of a long, high-beta straight section for production of neutrino beams into a lattice which is otherwise opti-

the fewer muon cooling stages will be required upstream with a considerable savings in complexity and expense.) The challenge, then, in the design of the storage ring is to extend the exceptionally large transverse and longitudinal emittances characteristic of the arc design to include the high-beta insertion. For reference, the muon beam entering the initial cooling stage has a normalized transverse emittance of $15,000 \pi \mathrm{mm}-\mathrm{mr}$ and a momentum spread of about $\pm 5 \%$.
\end{abstract} mized for transverse and longitudinal admittance. (The ring must be able to accept a very large emittance and large momentum spread muon beam.)

\section{INTRODUCTION}

A muon storage ring is also a source of neutrinos from muon decay. By recirculating the intense muon source beam, a muon storage ring is capable of efficient and intense neutrino production when the muons decay in long field-free regions (or straight sections). This paper discusses two $10-\mathrm{GeV}$ muon storage rings designed to optimize neutrinobeam production.

\section{DESIGN CONSIDERATIONS}

There are three principal considerations when optimizing a muon storage ring for neutrino beam production. The overriding consideration is to design a ring with an exceptionally large acceptance, both transversely and longitudinally, thereby reducing as much as possible the beam cooling required. This condition also presumes large-emitance muon beams can be effectively accelerated. The next two are specific to neutrino production. One is the conversion of a significant fraction of the stored muons into a directed beam of neutrinos; that is, the production straight must occupy a substantial portion of the ring circumference. Another is that the properties of the secondary neutrinos include the dynamics of the muons in the production straight. This implies that the neutrino beam will be colinear (at least to within the limits set by the decay kinematics) if the divergence of the parent muon beam is less than or comparable to the muon decay angle (which is $10 \mathrm{mr}$ at $10 \mathrm{GeV}$ ). Since the muon beam divergence in the decay straight can be controlled with a straightforward high-beta insert, the lattice parameters are not overly constrained. This allows the base lattice to be designed consistent with unusually large transverse and momentum admittances. (The intent here is that the greater the acceptance achieved in the storage ring, the

\footnotetext{
* Work supported by the U.S. Department of Energy under contract No. DE-AC02-76HO3000

$\dagger$ email: cjj@fnal.gov
}

\section{BASIC LATTICE DESIGN}

With long opposing straights, the natural layout of the storage ring is racetrack. Strong-focussing FODO cells are the best choice for the arcs because of their potential for large momentum acceptance (larger than $\pm 5 \%$ ) as compared with more complicated focussing structures. At 10$\mathrm{GeV}$, large-bore superconducting quadrupoles are recommended in order to maintain a strong gradient over the large aperture required to accomodate both the large transverse admittance and the large displacement of off-momentum orbits. The high-beta insert, on the other hand, is a weakerfocussing structure and must be carefully designed and matched to the arc to transmit the large range in momenta in addition to creating a parallel beam for neutrino production.

Table 1: Parameters of the large-momentum acceptance arc cells for a $10-\mathrm{GeV}$ muon storage ring

$\begin{array}{lcc} & \text { Initial } & \text { Later } \\ \text { intermagnet spacing }(\mathrm{m}) & 0.1451 & 0.2 \\ \text { dipole length }(\mathrm{m}) & 3.422 & 0.645 \\ \text { dipole bend }(\mathrm{rad}) & \pi / 18 & 0.174 \\ \text { dipole field }(\mathrm{T}) & 1.7 & 9 \\ \text { quadrupole length }(\mathrm{m}) & 0.4 & 0.387 \\ \text { arc quadrupole strength }\left(\mathrm{m}^{-2}\right) & 0.75 & 1.8 \\ \text { arc quadrupole poletip field }(\mathrm{T}) & >5 & 7.8 \\ \text { arc quadrupole radius }(\mathrm{cm}) & 20 & 13 \\ \text { cell phase advance }(\mathrm{deg}) & \approx 74 & \approx 90 \\ \text { horiz. sextupole strength }(\mathrm{T}) & 0 & 2.3 \\ \text { vert. sextupole strength }(\mathrm{T}) & 0 & 3.5\end{array}$

The initial design is simply constructed using two identical high-beta straights matched directly to the connecting arcs. Each arc is composed of six full FODO cells plus an extra half cell with cell properties given as the first example in Table 1. Two cells at each end of the arcs have reduced deflections to suppress dispersion in the two long straights. The arcs and dispersion suppression modules account for $44 \%$ of the ring which is $394 \mathrm{~m}$ in circumference. The high-beta straight for neutrino production was formed using an antisymmetric doublet quadrupole structure and repre- 

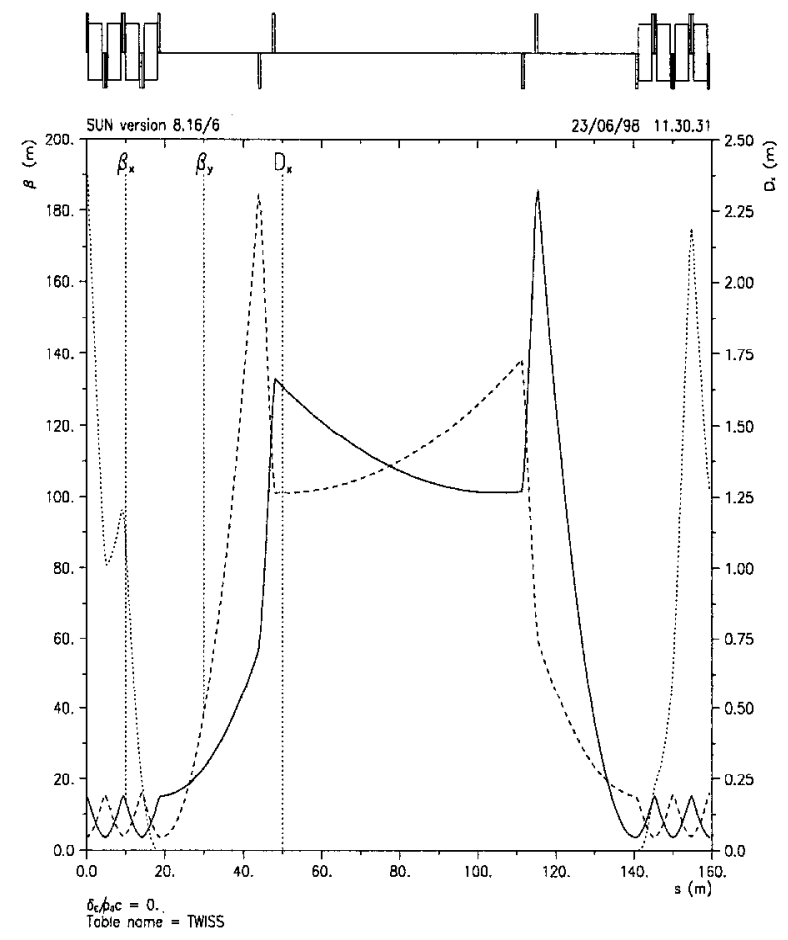

Figure 1: Initial design: The neutrino production region flanked by matching sections and dispersion-suppression cells

sents about $16 \%$ of the total ring circumference. The matching section between the arc and and the high-beta straight accounts for $12 \%$ of the total circumference and its increased divergence contributes to the backgrounds associated with neutrino detection. Fig. 1 shows the beta and dispersion functions for the high-beta region, flanking matching sections, and dispersion suppression cells. The rest of the circumference is occupied by a second long straight which is opposite to and presently identical to the production straight. This region will eventually be redesigned as a utility section to include injection. The periodicity of this ring is one.

The admittance of the initial ring design was calculated using a $\beta_{\text {max }}$ in the high-beta quadrupoles of $200 \mathrm{~m}$ (from Fig. 1), a quadrupole radial aperture of $20 \mathrm{~cm}$, and assuming $\mathrm{a} \pm 3 \sigma \mathrm{rms}$ beam size. These parameters give a normalized rms emittance of $2111 \mathrm{~mm}-\mathrm{mr}$; given that the upstream acceleration is not the limiting aperture. Errors have not been included in calculating this ideal aperture.

The momentum dependence of the initial lattice was studied and tracked using MAD[2]. The tune of the ring is $\nu_{x}=5.46$ and $\nu_{y}=5.65$. Tracking studies indicate that this fractional tune is near optimal and also that the momentum aperture is best when the $\mathrm{x}$ and $\mathrm{y}$ plane fractional tunes are close. (Although symmetric inserts were studied, it was more difficult to make the fractional tunes between the two planes close-at least in the present lattice version-and the dynamic aperture was smaller when tracked.) Although a closed orbit exists for a $d p / p$ of $-6.7 \%$ to $5.8 \%$, the orbit excursions were large at the low momenta and the betas too high at the high momenta. If an orbit cutoff of $\pm 15 \mathrm{~cm}$ and a $\beta_{\max }$ of $200 \mathrm{~m}$ is enforced, then the acceptable momentum range of the initial lattice design appears to be $-5.3 \%$ to $5 \%$.

\section{LATTICE IMPROVEMENTS}

Noting that the neutrino production straight is only $16 \%$ of the ring circumference, one of the first modifications to the design was to match the short FODO cells of the arc into much longer FODO cells to build up the high-beta production region. Use of a periodic unit allows almost complete flexibility in the length of the region. Additionally, peak beta values were reduced by a factor of two. The insertion was kept antisymmetric so that the periodicity of the ring remained one. The improvements in this later lattice allows for a production straight which can be easily varied from $\approx 22 \%$ of the total circumference for a $428 \mathrm{~m}$ ring to $\approx 40 \%$ for a $717 \mathrm{~m}$ circumference ring. In the initial design the malching sections are almost equivalent in length to the production straight ( $75 \%$ of), but in the later work, they fall to $30 \%$ the length of the production straight in the $428 \mathrm{~m}$ ring and $10 \%$ in the $717 \mathrm{~m}$ ring.

A significant problem with the initial design is that the tune changes by $\nu_{x}=5.82, \nu_{y}=6.01$ to $\nu_{x}=5.11, \nu_{y}=$ 5.28 over the accepted momentum range. Such a large tune spread unavoidably covers an integer or half-integer resonance; consequently substantial beam loss is expected. Therefore, another improvement in the later design was to insert sextupole correctors at the center of each quadrupole in the arcs and cancel most of the linear chromaticity. (In the calculation, the quadrupoles were divided in two and short, $0.2 \mathrm{~m}$ sextupole magnets inserted; hence their large poletip fields. It should be possible instead to insert sextupole correction coils along the length of the quadrupole at a reduced field.)

Not only were sextupoles correctors added, the arc cell was also redesigned to keep the off-momentum excursions under $\pm 8 \mathrm{~cm}$ and arc quadrupole apertures modest. Reducing off-momentum orbit excursions means shorter cells with stronger quadrupoles and shorter, stronger dipoles. The amount of bend per arc cell was left virtually unchanged so the number of cells per arc remains the same (7.5). Additionally, at the central momentum of $10 \mathrm{GeV}$, the arc quadrupole strengths were tuned to give to a near $\pi / 2$ phase advance per cell (keeping the focussing and defocussing quadrupoles equal strength). The new parameters of the arc FODO cells are included in Table 1 for comparison. It should be noted that the $9 \mathrm{~T}$ fields in the dipoles can be reduced to 6-7 $\mathrm{T}$ without compromising the basic lattice design or its properties; this requires adding 2 to 4 cells per arc. 


\section{RESULTS}
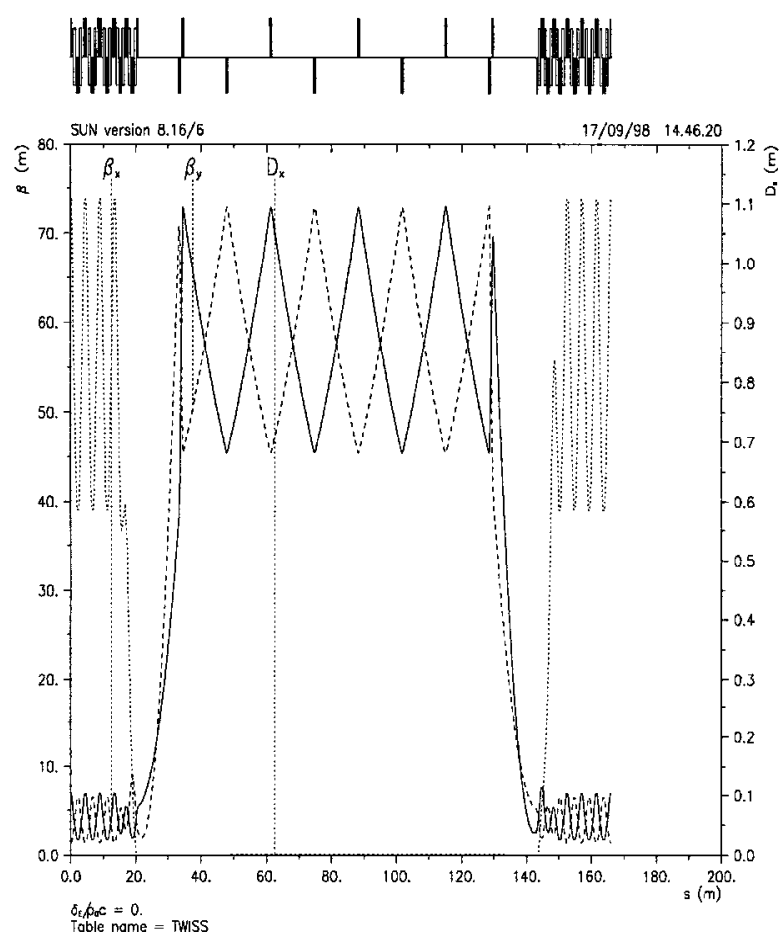

Figure 2: Later design: Lattice functions of the high-beta FODO cells for neutrino production flanked by matching sections, dispersion supression cells, and three arc modules.

\section{LATTICE PERFORMANCE}

The optimal dynamic aperture for both transverse and longitudinal was found to be near a fractional tune of $(2 n+$ 1) $\pi / 2$. In the latest lattice shown Fig. 2, the fractional tune of the horizontal plane was set near 0.25 and the vertical plane near 0.75.) This choice keeps off-momentum particles from approaching either integer or half-integer resonances using the weakest sextupole strengths. It also minimizes second-order chromatic effects. With this fractional tune, the normalized rms emittance accepted by the highbeta quadrupoles-assuming a $20 \mathrm{~cm}$ quadrupole radius, a $\beta_{\max }$ of $100 \mathrm{~m}$ and a $3 \sigma$ distribution-is about $4200 \mathrm{~mm}$ $\mathrm{mr}$. The transverse acceptance is limited by the high-beta quadrupole aperture and, fortunately, not by the nonlinear tuneshifts generated by the chromatic correction sextupoles.

With the sextupoles cancelling most of the linear chromaticity, the total tune swing is reduced from $\delta \nu_{x}=$ $0.71, \delta \nu_{y}=0.73$ to $\delta \nu_{x}=0.12, \delta \nu_{y}=0.16$. Further reduction of this tune swing is possible, but does not appear necessary and stronger sextupoles decrease the currently large on-momentum aperture.
At the rms emittance quoted (2111 mm-mr), the rms angle of the muon beam in the $63.3 \mathrm{~m}$-long production region (between the quadrupolc doublets) is less than $1 \mathrm{mr}$, or less than $1 / 10$ the natural opening angle of the neutrinos. It is therefore an insignificant contribution to the final divergence of the neutrino beam. However, the matching sections outside of the doublet contribute between 1 and $3 \mathrm{mr}$ over $33.2 \mathrm{~m}$, rising to $2-5 \mathrm{~m}$ over the 14 meters occupied by the high-beta quadrupole doublet.

In the later lattice, the rms angle of divergence, on average, in the production straight (assuming $4200 \mathrm{~mm}-\mathrm{mr}$ ) is about $1.3 \mathrm{mr}$ both horizontally and vertically, despite the increase in the transverse acceptance. There is a short burst of highly divergent beam, 14-17 mr, over the short distance $(\approx 1 \mathrm{~m})$ comprising the high-beta quadrupole doublet. However, the resulting background neutrinos should dissipate rapidly before the intended target. The divergence in the remaining $12-13 \mathrm{~m}$ in each matching section (representing over $90 \%$ of the length of this section), drops to $3-5 \mathrm{mr}$, which is similar to the previous design. Overall, the contribution of the matching sections to the neutrino beam is reduced by a factor of 2.5-7 from the initial design.

\section{DISCUSSION AND SUMMARY}

In the initial design, all quadrupoles must have large, 20$25 \mathrm{~cm}$ radial apertures to accomodate the large displacements $( \pm 15 \mathrm{~cm})$ of off-momentum orbits in the arcs and the $200 \mathrm{~m}$ high-beta peaks in the insert. This includes both arc and high-beta quadrupoles. In the arcs of the second design, the quadrupole apertures are smaller by about a factor of two due to the reduced orbit swing of off-momentum orbits. If needed, the strength and cell length can be adjusted to lower the poletip fields in both quadrupole and dipole components. Since the high-beta peak in the insert is lower by a factor of two, the transverse dynamic aperture of the second lattice is correspondingly larger.

To summarize, ideal lattices have been designed which successfully include a long, low-divergence, straight section for neutrino production without compromising an exceptional dynamic aperture in both transverse and momentum space. The production straights nominally occupy 20 $40 \%$ of the ring circumference indicating a large conversion of stored muons into useable neutrinos. Although a large dynamic aperture has been obtained, clearly, a realistic set of errors must be introduced into the tracking to determine to what extent this ideal aperture deteriorates.

Thanks to S. Ohnuma for useful suggestions on sextupoles

\section{REFERENCES}

[1] S. Geer, Fermilab-PUB-97/389 (hep-ph/9712290)

[2] H. Grote and F. Iselin, CERN publication CERN/SL-9013(AP), version 8.17 . 